\title{
Summit meeting may give French videotext international appeal
}

\section{Paris}

THE hugely successful, but as yet largely unexportable, French public videotext system, Teletel, turned a historic corner at the beginning of November when West Germany finally agreed to allow connection of Teletel's 'Minitel' terminals to the public telephone network. The agreement, signed by telecommunications ministers from France and West Germany at a summit meeting in Karlsruhe, followed a long dispute over standards.

West Germany's principal objection to Minitel - its internal modem — was gradually eroded during negotiations. German modem manufacturers (such as Siemens) wanted Minitels to use external (German) modems.

West Germany is now the first European country to connect directly into the French videotext network, effectively doubling the potential number of users. As part of the deal, French telecom (DGT) will team up with its German equivalent, Deutsche Bundespost, in the design of a new telephone workstation and the construction of a fibre-optic link between Mulhouse and Karlsruhe.

Since the first field trials between 1980 and 1983, Teletel has attracted almost 3 million subscribers, whereas Britain's Prestel - which pioneered videotext and may yet prosper, see page 410 - still has only 78,000 and West Germany's Bildschirmtext (BTX), 60,000. The French DGT learned from Prestel's slow take-off that the public were reluctant to invest in the hardware needed to connect into the network, particularly in the beginning, when few services were on offer. Similarly, with few subscribers, it was hard to persuade advertisers and suppliers of valued-added services of the potential.

The DGT therefore decided to create a large pool of Minitel users at the outset, by distributing free terminals (comprising internal modem, screen and keyboard), with free access to an electronic directory service (EDS), instead of the telephone book. EDS, the only Teletel service offered by DGT, is still the most called, registering 24 million calls and 900,000 connection hours in June this year.

The success of Teletel is due also to a kiosk system of charging for services, whereby suppliers take part of a higher telephone charge, instead of demanding a subscription fee, which often discourages casual users. A flourishing range of online bulletin-boards has grown up precisely because callers remain anonymous.

The modular conception of the underlying communications network also makes it efficient and easily expandable. While user terminals are connected to the ordi- nary public-switched telephone network, host computers supplying Teletel services are connected to the Transpac packetswitched data network. The link between the two is provided by access points around the country. As Transpac provides gateways to foreign telecom networks around the world, Minitel may, in theory, be exploited abroad.

Here, however, comes the rub. Teletel databases are of interest to foreign users, while the large French user network is attractive for foreign service suppliers. The basic Minitel terminals, manufactured by Telic Alcatel, Matra and Radiotechnique, are, however, too rudimentary to warrant substantial export orders. In five years, only 100,000 terminals have been sold abroad - notably to Spain $(30,000)$, Switzerland (6,000), Canada $(30,000)$, the United States, Belgium and Brazil. These terminals are usually used as part of an internal network, not to connect with French Teletel.

Differences in telecom standards between France and other countries have also limited attempts to export Minitel technology. The standard Telic Alcatel/ Radiotechnique Minitel uses an outdated 'V23' modem that does not conform with rapid data-transmission standards in the United States and some other countries. So multistandard 'de-luxe' Minitels, including a joint Teletel/Prestel version, have been developed by French manufacturers, with approval in 14 countries.

However, the growing number of Minitel emulation cards for personal computers may nip in the bud the expected extra business for Minitel's French makers while cutting down DGT's profits from Teletel calls. These cards make use of hardware already purchased by the user, while adding a modem. Clever software uses the computer's peripherals to shortcut the time-wasting advertising pages and inefficient Minitel screen, which usually prolong EDS calls beyond the first $3 \mathrm{~min}$ utes for which they are free. Peter Coles

\section{NSF to spend \$14 million on MERIT supercomputer network}

Washington

MERIT, a consortium of Michigan universities, will soon be taking over the operation of NSFNET, a high-speed, nationwide backbone network that links regional computer networks and supercomputer centres. The National Science Foundation (NSF) announced last week that it is giving MERIT a grant of $\$ 14$ million for five years to run the network. But also important to MERIT's plans to expand and upgrade NSFNET are commitments of financial support and support in kind from IBM, MCI Communications Corporation and the state of Michigan.

Computer networks have proliferated in the United States, creating protocol nightmares for internet communications. NSFNET is an attempt to simplify matters.

Sending data through a network is accomplished using packet switching; data files are broken into individual 'packets', routed independently through the network in the most efficient manner possible and then reassembled at their destination. The speed of the network is determined by how fast the transmission lines connecting the nodes can transmit data, and how clever the algorithms are for routing packets efficiently through the network.

MERIT's plans for NSFNET call for transmission lines that can carry data at 1.5 megabits per second to be in use by June 1988. MCI will provide long-distance lines and will help underwrite the cost of local telephone company connections.

MCI's contribution is reckoned to be worth approximately $\$ 7$ million. For its part, IBM will provide switching hardware, as well as software for network management and informational services. Depending on how it is calculated, IBM's input to NSFNET is valued at between $\$ 10$ and $\$ 30$ million. The state of Michigan is contributing $\$ 5$ million to the project.

Stephen Wolff, director of NSF's division of networking and communication research and infrastructure, sees NSFNET as an opportunity for industry to get a head start on what he expects will be a proliferation of high-speed data networks in the future. He also hopes NSFNET will help NSF to take the lead in making sense of the current jumble of networks used by federal agencies.

NSFNET will be operational next summer using 1.5-megabit lines, but MERIT plans to develop the switching capability to handle lines that can transmit data 30 times faster. Such high-speed networking capabilities open new possibilities in real-time communications. Higherspeed networking also will mean greater remote access to national supercomputer centres. NSF now supports five such centres: Cornell University, Princeton University, the University of Illinois at Champaign-Urbana, the University of Pittsburgh and the University of California at San Diego. Each will be a node in the NSFNET backbone together with the supercomputer centre at the National Center for Atmospheric Research in Boulder, Colorado. 